\title{
Van Allen Probes observation of plasmaspheric hiss modulated by injected energetic electrons
}

\author{
Run Shi ${ }^{1}$, Wen Li $^{1}$, Qianli Ma ${ }^{2,1}$, Seth G. Claudepierre ${ }^{3}$, Craig A. Kletzing ${ }^{4}$, William S. Kurth ${ }^{4}$, \\ George B. Hospodarsky ${ }^{4}$, Harlan E. Spence ${ }^{5}$, Geoff D. Reeves ${ }^{6}$, Joseph F. Fennell ${ }^{3}$, J. Bernard Blake ${ }^{3}$, \\ Scott A. Thaller ${ }^{7}$, and John R. Wygant ${ }^{7}$ \\ ${ }^{1}$ Center for Space Physics, Boston University, Boston, Massachusetts, USA \\ ${ }^{2}$ Department of Atmospheric and Oceanic Sciences, University of California, Los Angeles, Los Angeles, California, USA \\ ${ }^{3}$ Space Science Department, The Aerospace Corporation, El Segundo, California, USA \\ ${ }^{4}$ Department of Physics and Astronomy, University of Iowa, Iowa City, Iowa, USA \\ ${ }^{5}$ Institute for the Study of Earth, Oceans, and Space, University of New Hampshire, Durham, New Hampshire, USA \\ ${ }^{6}$ Space Science and Applications Group, Los Alamos National Laboratory, Los Alamos, New Mexico, USA \\ ${ }^{7}$ School of Physics and Astronomy, University of Minnesota, Twin Cities, Minneapolis, Minnesota, USA
}

Correspondence: Run Shi (runs@bu.edu)

Received: 4 January 2018 - Discussion started: 17 January 2018

Revised: 30 March 2018 - Accepted: 3 April 2018 - Published: 23 May 2018

\begin{abstract}
Plasmaspheric hiss was observed by Van Allen Probe $\mathrm{B}$ in association with energetic electron injections in the outer plasmasphere. The energy of injected electrons coincides with the minimum resonant energy calculated for the observed hiss wave frequency. Interestingly, the variations in hiss wave intensity, electron flux and ultra low frequency (ULF) wave intensity exhibit remarkable correlations, while plasma density is not correlated with any of these parameters. Our study provides direct evidence for the first time that the injected anisotropic electron population, which is modulated by ULF waves, modulates the hiss intensity in the outer plasmasphere. This also implies that the plasmaspheric hiss observed by Van Allen Probe B in the outer plasmasphere $(L>\sim 5.5)$ is locally amplified. Meanwhile, Van Allen Probe A observed hiss emission at lower $L$ shells $(<5)$, which was not associated with electron injections but primarily modulated by the plasma density. The features observed by Van Allen Probe A suggest that the observed hiss deep inside the plasmasphere may have propagated from higher $L$ shells.
\end{abstract}

\section{Introduction}

Plasmaspheric hiss plays an important role in the loss of energetic electrons within the plasmasphere and in high-density plumes (Lyons et al., 1972; Lyons and Thorne, 1973; Albert, 2005; Meredith et al., 2007, 2009; Summers et al., 2008; Ni et al., 2013; Breneman et al., 2015; Li et al., 2015a; Ma et al., 2016). However, the generation mechanisms of plasmaspheric hiss remain under active research. Three mechanisms have received the most intense attention to explain the generation of plasmaspheric hiss, including in situ growth of waves (Thorne et al., 1979; Church and Thorne, 1983), lightninggenerated whistlers (Green et al., 2005), and whistler mode chorus waves as an "embryonic source" (Bortnik et al., 2008, 2009; Chen et al., 2012a, b). Although wave power above 2$3 \mathrm{kHz}$ from lightning-generated whistlers shows some correlation with hiss waves (Green et al., 2005), the waves below $1 \mathrm{kHz}$, which contain the majority of hiss wave power, are independent of the lightning flash rate (Meredith et al., 2006). The in situ growth of waves inside the plasmasphere was shown to be inadequate to account for the observational level ( $20 \mathrm{~dB}$ ) (Huang et al., 1983); in response, Church and Thorne (1983) suggested that an "embryonic source" is required to lead to the observed wave intensity. Recent studies based on ray tracing simulation (Bortnik et al., 2008) have demonstrated that chorus waves from the distant magneto- 
sphere can propagate into the plasmasphere and act as an embryonic source for the hiss wave generation. Furthermore, ray tracing simulations (Chen et al., 2012a) suggested that the majority of hiss formation is caused by chorus emission originating within $\sim 3 R_{\mathrm{E}}$ from the plasmapause. This model has successfully explained the observed frequency spectrum and spatial distribution of the observed hiss over the typical hiss frequency range from $100 \mathrm{~Hz}$ to several $\mathrm{kHz}$. A number of observational studies (Bortnik et al., 2009; Wang et al., 2011; Meredith et al., 2013; Li et al., 2015b) have shown good correlations between chorus and plasmaspheric hiss and suggested that chorus plays an important role in hiss wave intensification.

The Van Allen Probes mission recently detected unusual low-frequency hiss emissions with wave power extending well below $100 \mathrm{~Hz}$ (Li et al., 2013). The low-frequency hiss was demonstrated to cause more efficient loss of high-energy electrons (from $\sim 50 \mathrm{keV}$ to a few $\mathrm{MeV}$ ) due to its stronger pitch angle scattering rates compared to normal hiss (Ni et al., 2014; Li et al., 2015a). Such a low-frequency hiss is unlikely to be a result of propagation of chorus waves from a more distant region because embryonic chorus waves at the same frequency (Bortnik et al., 2008) would need to originate from unrealistically high $L$ shells (Li et al., 2015b). Therefore, these low-frequency hiss waves were suggested to be generated in the outer plasmasphere on the dayside through local amplification (Li et al., 2013; Chen et al., 2014; Shi et al., 2017).

Hiss intensity modulation is often driven by the variation in background plasma density either through local amplification or wave propagation (Chen et al., 2012c), and the modulation of hiss by other factors may easily be suppressed by the effect of the plasma density. Therefore, observations showing a direct correlation between hiss emission and electron flux are still very limited. In fact, electron fluxes of energetic electrons (tens to hundreds of $\mathrm{keV}$ ) can be modulated by ultra low frequency (ULF) waves. A typical modulation is caused by drift resonance (Southwood and Kivelson, 1981). Zong et al. (2009) showed an interesting event of energetic electron modulation by shock-induced ULF waves. More recently, Claudepierre et al. (2013) presented observations of electron drift resonance with the fundamental poloidal mode of ULF waves based on Van Allen Probes measurements. The energy dependence of the amplitude and phase of the electron flux modulations provided strong evidence for such an interaction. The peak electron flux modulations occurred over 5-6 wave cycles at energies $\sim 60 \mathrm{keV}$. The drift resonance between electrons and ULF waves has been extensively studied both theoretically and observationally based on Van Allen Probes data (Dai et al., 2013; Hao et al., 2014; Chen et al., 2016; Zhou et al., 2015, 2016; Li et al., 2017). Such a modulation of energetic electrons may modulate hiss emissions by varying the electron flux and pitch angle anisotropy, which could potentially affect the local growth rates of hiss waves, but the observational evidence has not been reported yet. In this study, we report on a modulation of hiss wave intensity and injected electron flux due to ULF waves observed by Van Allen Probe B near the dayside, providing clear evidence that the hiss emission was generated through local amplification in the outer plasmasphere.

\section{Data and methodology}

The Van Allen Probes mission comprises two identical spacecraft (probes A and B) in near-equatorial orbits with an altitude of $\sim 600 \mathrm{~km}$ at perigee and geocentric distance of $\sim 5.8 R_{\mathrm{E}}$ at apogee (Mauk et al., 2012). The Electric and Magnetic Field Instrument Suite and Integrated Science (EMFISIS) suite on board Van Allen probes A and B includes a magnetometer and a "waves" instrument (Kletzing et al., 2013). The DC magnetic field is measured by the magnetometer, and the survey mode of the waveform receiver (WFR) provides the power spectral density from $10 \mathrm{~Hz}$ to $12 \mathrm{kHz}$ at $6 \mathrm{~s}$ time resolution. Plasma density can be either calculated based on the upper hybrid resonance frequency extracted from the high frequency receiver (HFR) data (Kurth et al., 2015) or be inferred from the spacecraft potential measured by the electric field and waves (EFW) instrument (Wygant et al., 2013). We inferred plasma density profiles based on the measurements from both instruments in the present study to obtain accurate plasma density values with high time resolution. High-resolution electron flux measurements over the energy range of $\sim 30 \mathrm{keV}$ to $4 \mathrm{MeV}$ are provided by the magnetic electron ion spectrometer (MagEIS) instrument (Blake et al., 2013; Spence et al., 2013). We used the level 3 MagEIS dataset, which includes particle pitch angle distribution, in this study to evaluate the electron distribution responsible for the hiss wave generation.

\section{Observational results}

A hiss intensification event modulated by electron injection was observed by Van Allen Probe B during 20:0022:00 UT on 12 January 2014, as shown in Fig. 1. The satellite was located on the dayside and remained inside the plasmasphere, indicated by the high plasma density (Fig. 1f). The main power of the hiss emission (Fig. 1b and c) resided below the lower hybrid resonance frequency (white dash-dotted line in Fig. 1b) and $100 \mathrm{~Hz}$ (white dashed line in Fig. 1c) and intensified following the increase in the AE index (geomagnetic auroral electrojet index; Fig. 1a). Figure 1e presents the magnitude of the background magnetic field. The spinaveraged electron flux (Fig. 1g) exhibited modulations with a period of about $6 \mathrm{~min}$. There is also a variation in the electron pitch angle anisotropy (Fig. 1h) although it is not as clear as the modulations of electron flux. The electron anisotropy is calculated based on Chen et al. (1999). The black lines in Fig. $1 \mathrm{~g}$ and $\mathrm{h}$ show the calculated minimum electron resonant energy for the first-order cyclotron resonance with parallel- 


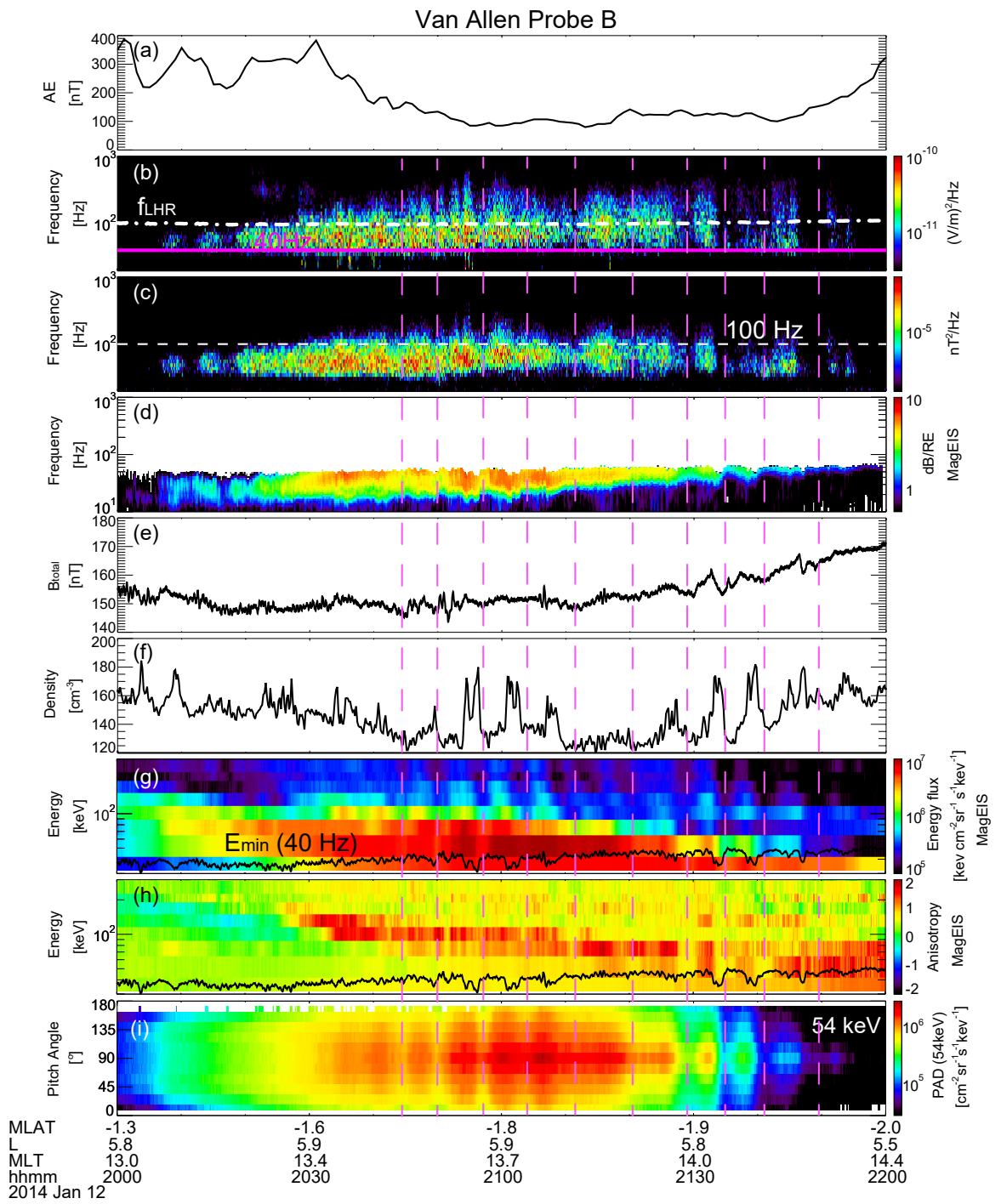

Figure 1. Plasmaspheric hiss modulation caused by injected electrons observed by Van Allen Probe B from 20:00 to 22:00 UT on 12 January 2014. (a) AE index; frequency-time spectrogram of (b) wave electric field and (c) wave magnetic field spectral density in the WFR channel; (d) frequency spectrum of convective linear wave growth rates; (e) background magnetic field intensity; (f) calibrated plasma density based on EFW and EMFISIS; (g) spin-averaged electron flux measured by MagEIS; (h) electron pitch angle anisotropy; (i) pitch angle distribution of electrons at $54 \mathrm{keV}$. The white dash-dotted line in (b) represents the lower hybrid resonance frequency $\left(f_{\mathrm{LHR}}\right)$. The magenta line in (b) indicates $40 \mathrm{~Hz}$. The white dashed line in (c) indicates $100 \mathrm{~Hz}$. The black lines in (g, h) represent the minimum resonant energy of electrons interacting with the waves at $40 \mathrm{~Hz}$. The dashed vertical lines mark the modulation of the electron flux at $54 \mathrm{keV}$ (i).

propagating right-hand polarized waves at a frequency of $40 \mathrm{~Hz}$ (magenta line in Fig. 1b). As shown in Fig. 1g, the minimum resonant energy captures the main energy of injected electrons. Figure 1i shows the electron pitch angle distribution at $54 \mathrm{keV}$, which exhibits a pronounced modulation. The vertical dashed lines present the minima of the electron fluxes at $54 \mathrm{keV}$. Figure 1d illustrates the convective linear growth rates for parallel-propagating whistler mode waves that were calculated using the electron distribution measured by MagEIS based on the equations of Summers et al. (2009). The modulation of linear growth rate appears to correlate well with the observed hiss wave spectral intensity with a period of several minutes.

Changes in the background magnetic field, plasma density and the injected electron distribution (flux and pitch angle anisotropy of resonant electrons) could potentially be responsible for the hiss wave growth. Since the variation of the background magnetic field is small $(\sim 4 \mathrm{nT})$ compared to the median value $(\sim 150 \mathrm{nT})$, the effect of background magnetic field on the wave growth rate is likely to be insignificant compared to the effects of plasma density and electron injection. To distinguish the roles of these two effects in the 


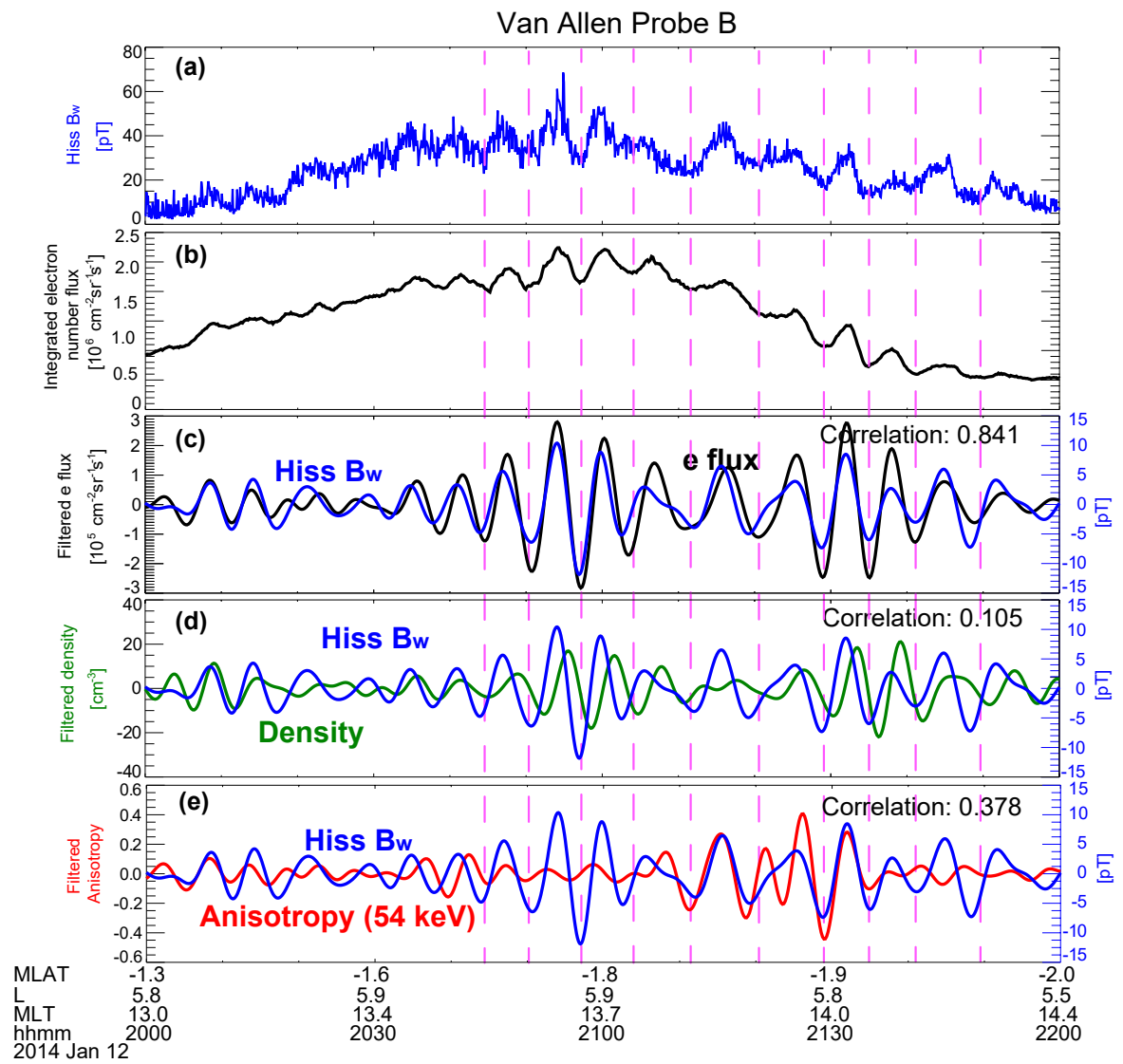

Figure 2. (a) Integrated hiss intensity from 20 to $1000 \mathrm{~Hz}$; (b) integrated spin-averaged electron flux from 30 to $200 \mathrm{keV}$; (c) filtered integrated electron number flux (black) and filtered magnetic wave intensity of hiss (blue); (d) filtered plasma density (green) and filtered magnetic wave intensity of hiss (blue); (e) filtered pitch angle anisotropy (red) and filtered magnetic wave intensity of hiss (blue). The vertical dashed lines depict the same times as those in Fig. 1.

local wave amplification, we compared the hiss wave amplitude with spin-averaged electron flux and plasma density. The hiss wave amplitude integrated from 20 to $1000 \mathrm{~Hz}$ is shown in Fig. 2a. Figure 2b presents the spin-averaged electron flux integrated over the energy range from 30 to $200 \mathrm{keV}$. The vertical dashed lines in Fig. 2 depict the same times as in Fig. 1.

Figure $2 \mathrm{c}$ shows the comparison between the filtered electron flux (black) over $1.5-4 \mathrm{mHz}$ and the filtered hiss wave intensity (blue) over $1.5-4 \mathrm{mHz}$. It suggests that the hiss intensity is well correlated with the variation of the electron flux. The correlation coefficient between the filtered electron flux and the filtered hiss wave intensity in the time period from 20:00 to 22:00 UT is 0.841 . The satellite was located at a magnetic latitude of -1.3 to $-2.0^{\circ}$, which was near the source region where local wave amplification typically occurs, and this is probably why hiss intensity and electron flux exhibit a remarkable correlation.

In the present hiss modulation event, the filtered background plasma density (green line in Fig. 2d) is not well correlated with the filtered wave intensity (with a correla- tion coefficient of 0.105 ), especially during the period from 20:45 to 21:40 UT. This suggests that the variation in plasma density plays an insignificant role in the modulation of hiss wave intensity during this event. To investigate the sole effect of density on hiss intensity, we also calculated the correlation coefficient between the non-filtered hiss wave intensity and the non-filtered plasma density which even shows a slight anti-correlation with a coefficient of $\sim-0.483$.

The comparison between the filtered electron pitch angle anisotropy at $54 \mathrm{keV}$ and filtered wave intensity is shown in Fig. 2e. Although a correlation coefficient of 0.378 indicates a certain correlation between these two parameters, it is much lower than the correlation between the hiss wave intensity and electron flux (0.841). Therefore, we suggest that the variation of electron pitch angle anisotropy play a less important role in hiss intensity modulation compared to the variation in electron flux.

The electron flux variation observed by Van Allen Probe B may be caused by ULF wave modulation since they have similar time periods. Figure 3 shows the variation of electron fluxes at different energy channels observed by both Van 

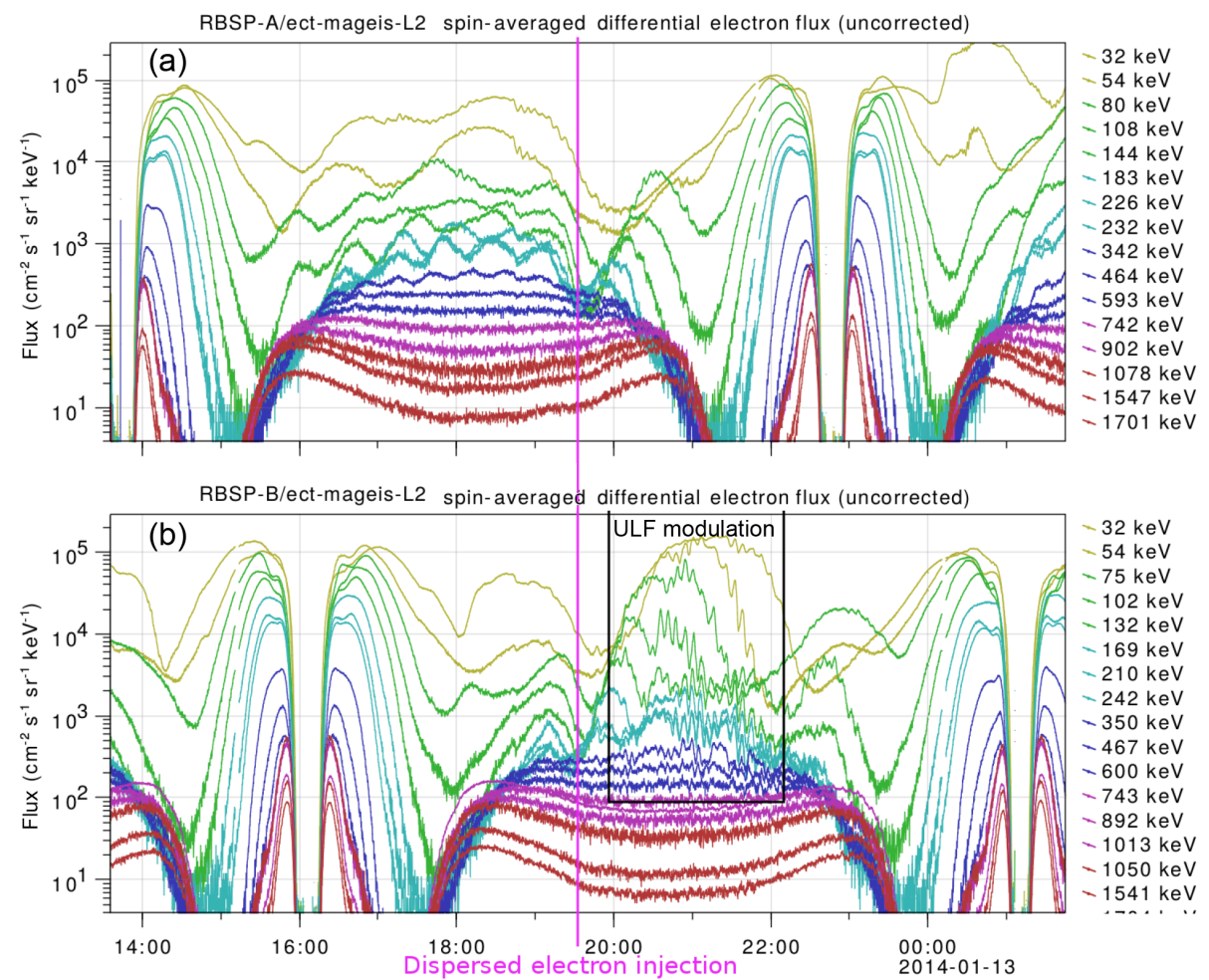

Figure 3. Variation of electron fluxes at different energies observed by Van Allen Probe A (a) and Van Allen Probe B (b). In (b), the modulation of electron fluxes was observed by Van Allen Probe B between 20:00:00 and 22:00:00 UT in association with ULF waves, and the dispersed electron injection was observed at $\sim$ 19:30:00 UT.

Allen Probe A (panel a) and Van Allen Probe B (panel b). At $~ 19: 30$ UT, both probes, especially Van Allen Probe B, observed intense electron injections. Between 20:00 and 22:00 UT, the energetic electron fluxes observed by probe B are modulated at most energy channels, with a time period of several minutes in the same frequency range as typical ULF waves (Pc4-5).

Figure 4 is the summary of the Pc4-5 ULF waves from Van Allen Probe B during the time interval of interest (20:0022:00 UT). Dynamic spectrograms of the ULF wave powers are shown for the three components of the magnetic field (in the mean field-aligned, geocentric solar magnetospheric, GSM, coordinates) along with the y component of the electric field in modified geocentric solar elliptic (MGSE) coordinate. Band-pass filtered time series $(1.5-4 \mathrm{mHz})$ are shown below for each dynamic spectrogram. The parallel magnetic field $\left(B_{\text {para }}\right)$ and $y$ component electric field in MGSE coordinate $\left(E_{y}\right)$ have a similar frequency peak at $\sim 2.6 \mathrm{mHz}$. The wave spectra of the $E_{y}$ and $B_{\text {para }}$ components suggest that the compressional mode and shear mode are likely coupled.

The correlation of the ULF waves and the energetic electron fluxes at different energy channels is shown in Fig. 5. Figure 5a illustrates the filtered $E_{y}$ component of the electric field between 1.5 and $4 \mathrm{mHz}$. Since Van Allen Probe B is near noon, the $E_{y}$ component approximately represents the electric field in the azimuthal direction. Band-pass filtered electron fluxes normalized by unperturbed levels at different energy channels are shown in Fig. 5b. The vertical black lines indicate the minima of the $E_{y}$ component. The electron fluxes at various energies show a modulation period which is very similar to that of $E_{y}$. Besides, these fluxes exhibit an energy-dependent phase shift with respect to $E_{y}$. The phase of the electron flux oscillations with respect to $E_{y}$ is closest to $180^{\circ}$ out of phase at $\sim 466 \mathrm{keV}$. At lower energies, the phase of peak electron fluxes relative to the $E_{y}$ minimum varies but is not $180^{\circ}$ out of phase. For the observed modulating hiss, the minimum resonant energy is tens of $\mathrm{keV}$ (Fig. 1), and thus the electron flux at energy below $100 \mathrm{keV}$ plays a dominant role in hiss amplification. Although these low-energy electrons (30-100 keV) are not exactly in drift resonance with the observed ULF waves, their modulation is highly relevant to the presence of ULF waves. These lowenergy electrons may be accelerated by the ULF waves during the first half cycle and then decelerated so that there is no total energy gain. This mechanism was also demonstrated in the drift-resonance theory in which the peak electron fluxes should have a $180^{\circ}$ energy shift (Southwood and Kivelson, 1981).

Meanwhile, Van Allen Probe A detected hiss emissions in a similar frequency range as shown in Fig. 6. During this time period, Van Allen Probe A was located at lower $L$ shells $(2.6<L<5.3)$ and later magnetic local times 

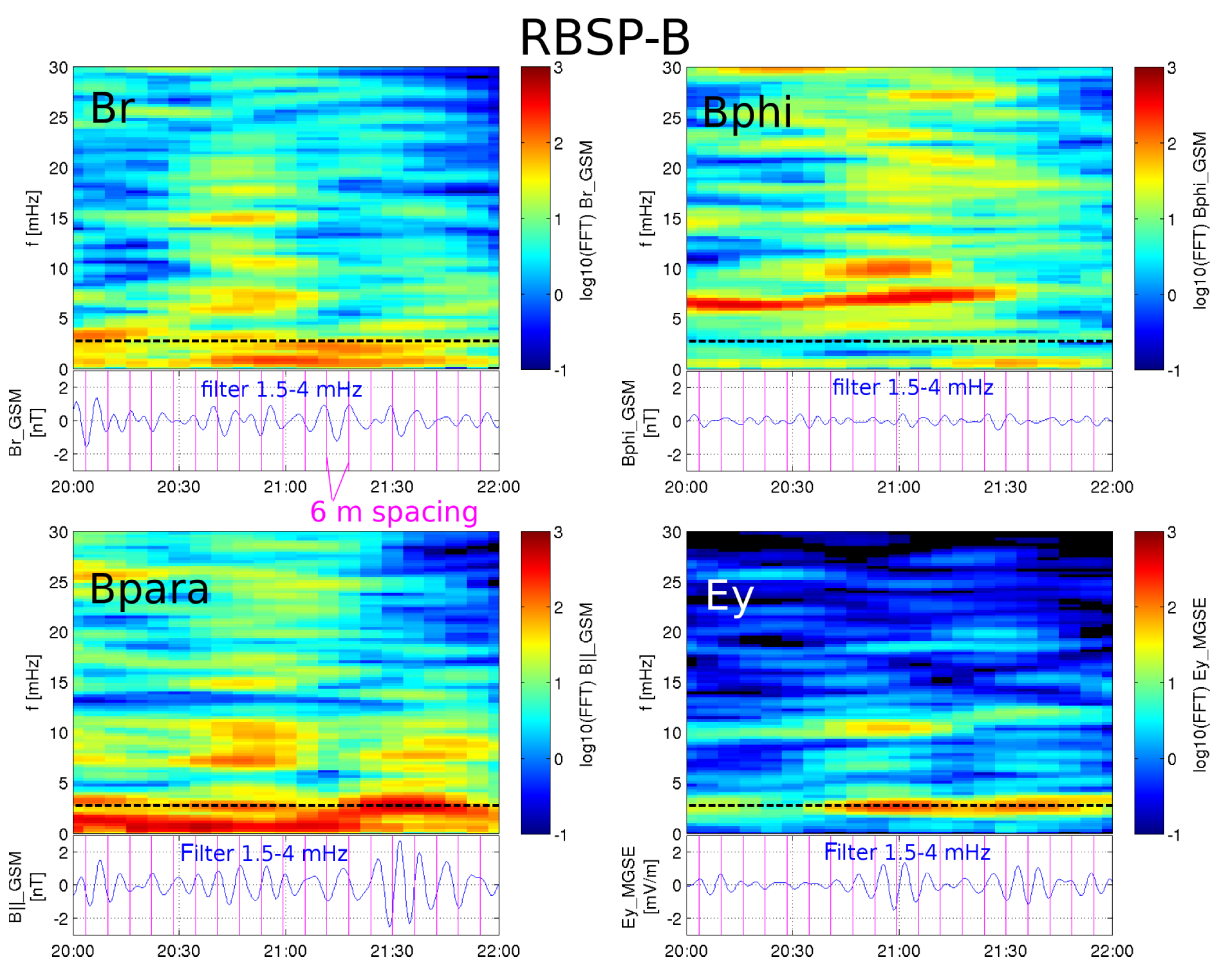

Figure 4. Summary of the Pc4-5 ULF wave frequency spectra from Van Allen Probe B during the time interval of interest (20:00-22:00 UT). Dynamic spectrograms are shown for the three components of the magnetic field (in the mean field-aligned, GSM coordinates) along with the y component of the electric field in MGSE coordinate. Band-pass filtered time series $(1.5-4 \mathrm{mHz})$ are shown below each dynamic spectrogram. The black dashed lines indicate the frequency at $\sim 2.6 \mathrm{mHz}$.

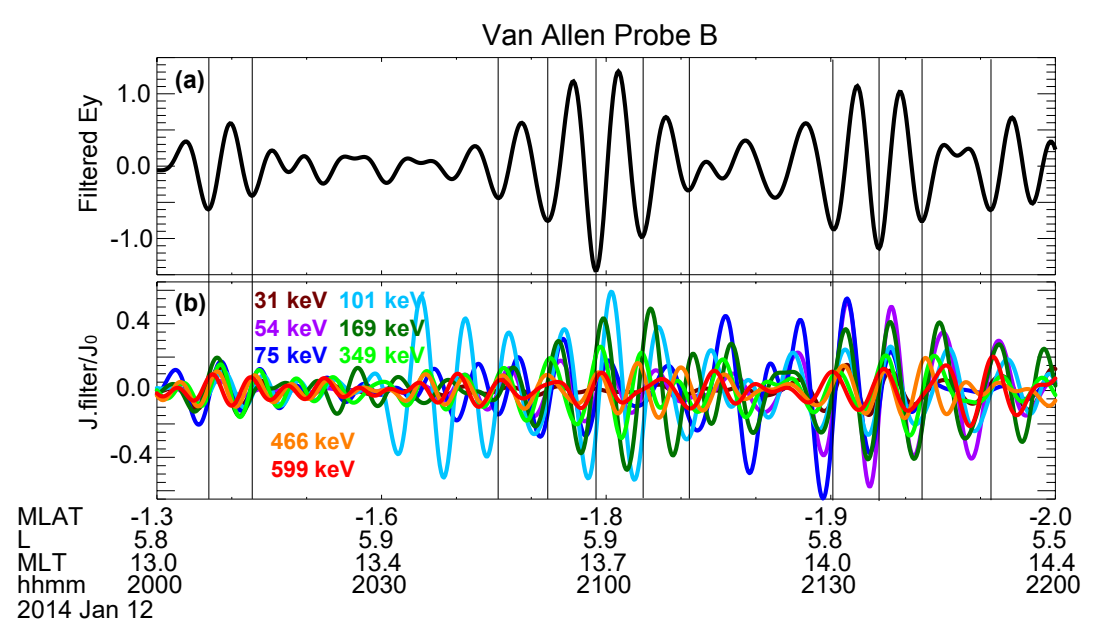

Figure 5. The correlation of the filtered (1.5-4 mHz) $E_{y}$ component of ULF waves (a) and the energetic electron fluxes at different energy channels (b). The electron fluxes show the modulation in the similar period to that of $E_{y}$, but exhibit an energy-dependent phase shift with respect to $E_{y}$.

$(14.9<$ MLT < 18.0). The hiss intensity also exhibited modulation in electric and magnetic field, as shown in Fig. 6b and $\mathrm{c}$, respectively. However, different from the observation by probe $\mathrm{B}$, the hiss intensity is dominantly modulated by the variation in the plasma density. Figure $6 \mathrm{~d}$ shows the density profile obtained from EMFISIS (black) and EFW (red). Ex- amples of evident modulations by variation in plasma density are highlighted with grey blocks. According to ray tracing simulation (Chen et al., 2012c), the hiss waves tend to propagate to the region with higher density resulting in higher wave intensity. Figure $6 e$ and $f$ show the spin-averaged electron flux and pitch angle anisotropy based on MagEIS data 


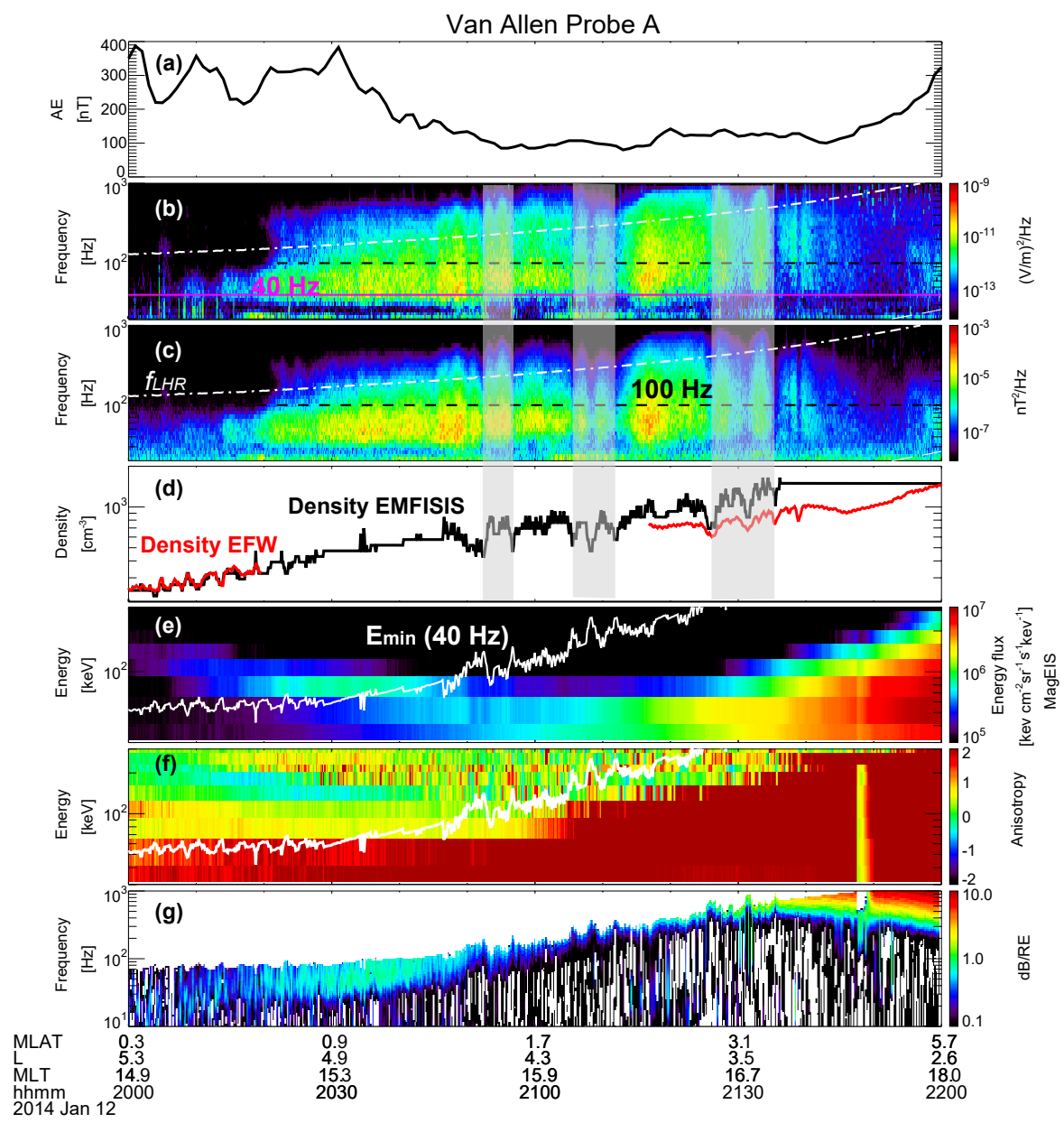

Figure 6. The observation of waves and electron fluxes by Van Allen Probe A during the same period as that in Fig. 1. (a) AE index; (b) frequency-time spectrogram of wave electric field and (c) wave magnetic spectral density in the WFR channel; (d) plasma density obtained by EFW (red) and EMFISIS (black); (e) spin-averaged electron flux measured by MagEIS; (f) electron pitch angle anisotropy; (g) convective wave growth rates. Grey block areas indicate the intervals of hiss modulation by variation of plasma density. The magenta line in (b) indicates $40 \mathrm{~Hz}$. The black dashed line in (c) indicates $100 \mathrm{~Hz}$. The white lines in (e, f) represent the minimum resonant energy of electrons for the waves at $40 \mathrm{~Hz}$.

and the white lines are the minimum resonant energy corresponding to a frequency of $40 \mathrm{~Hz}$ (Fig. 6b). There is no clear correlation between the hiss intensity and electron flux, suggesting that the modulations are mainly caused by the plasma density variation. We also calculated the convective linear growth rates for parallel-propagating whistler mode waves as shown in Fig. 6g. The growth rate profile shows little correlation with that of the observed hiss intensity, indicating that these waves are not locally excited.

Figure 7 illustrates the comparison of hiss wave frequency spectra observed by Van Allen probes A (Fig. 7a-b) and B (Fig. 7c-d). At the beginning of the emission around 20:20 UT, the hiss wave intensity as a function of frequency observed by Van Allen Probe A presents a minimum at $\sim 200 \mathrm{~Hz}$ (indicated by the white arrows in Fig. 7a and b). This feature is similar to the observation by Van Allen Probe
B (Fig. 7c and d), where the modulation of hiss wave power below $100 \mathrm{~Hz}$ is correlated with the calculated wave growth rate (Fig. 1d) based on the observed electron distribution. The hiss wave frequency spectra and structures observed by probe $\mathrm{A}$ are similar to those observed by probe $\mathrm{B}$, but the energy spectra of energetic electrons are significantly different. Therefore, the hiss emission observed by probe A may be the result of wave propagation from the source region in the outer plasmasphere and further modulated by the local plasma density variation.

\section{Summary and discussion}

We report clear evidence of local amplification of plasmaspheric hiss observed by Van Allen Probe B in the post-noon sector of the outer plasmasphere. The minimum resonance 


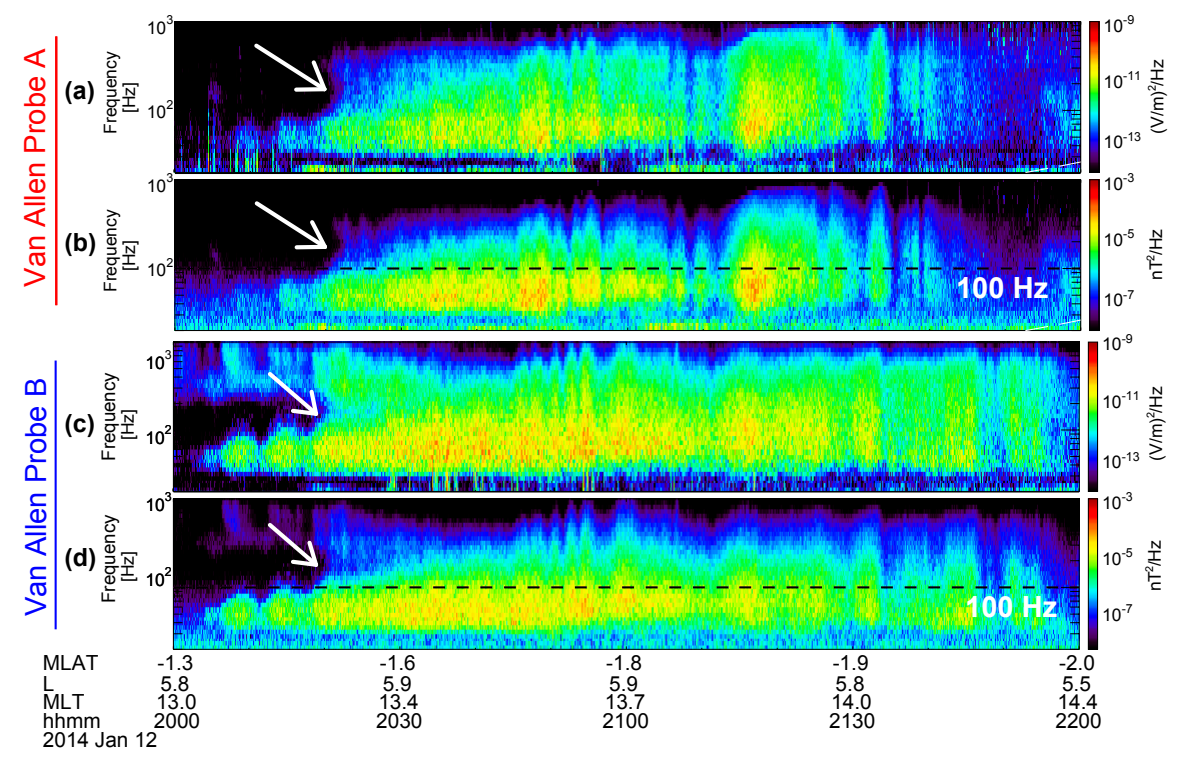

Figure 7. The wave electric (a) and magnetic (b) spectral density observed by Van Allen Probe A and the wave electric (c) and magnetic (d) spectral density from Van Allen Probe B. Note that at the beginning of the emissions around 20:20 UT, the hiss wave intensity as a function of frequency presents a minimum at $\sim 200 \mathrm{~Hz}$ (white arrows) for the observations from both Van Allen probes A and B.

energy calculated for the observed hiss wave frequency is consistent with the energy of injected electrons. The hiss wave intensity was modulated by the injected energetic electrons, which were modulated by ULF waves. In the meantime, Van Allen Probe A also observed similar hiss emissions at lower $L$ shells, which is probably due to the propagation from the source region in the outer plasmasphere. Different from the observation by probe B, the hiss wave intensity observed by probe A is predominantly affected by the background plasma density. The modulation of hiss intensity by plasma density could be due to the effect of ray focusing at a high-density region during propagation (Chen et al., 2012c).

Figure 8 summarizes the processes discussed in this study. The injected energetic electrons with energies of tens to hundreds of $\mathrm{keV}$ drift from the nightside to the dayside in the outer plasmasphere. Simultaneously, the ULF waves modulate the energetic electron fluxes. The modulated energetic electrons then lead to the modulation of the hiss intensity via local amplification. These features were all well captured by Van Allen Probe B. During the same time period, probe A at a later MLT and lower $L$ shell observed hiss emissions which may originate from the source region in the outer plasmasphere.

Chorus waves which are intense coherent electromagnetic emissions exhibiting discrete rising or falling tones are believed to be generated through cyclotron resonance with anisotropic electrons (Kennel and Petschek, 1966; Anderson and Maeda, 1977; Meredith et al., 2001; Li et al., 2009). It has been shown that ULF waves can modulate chorus intensity by modulating the background magnetic field and/or plasma density which affect the number of energetic elec-

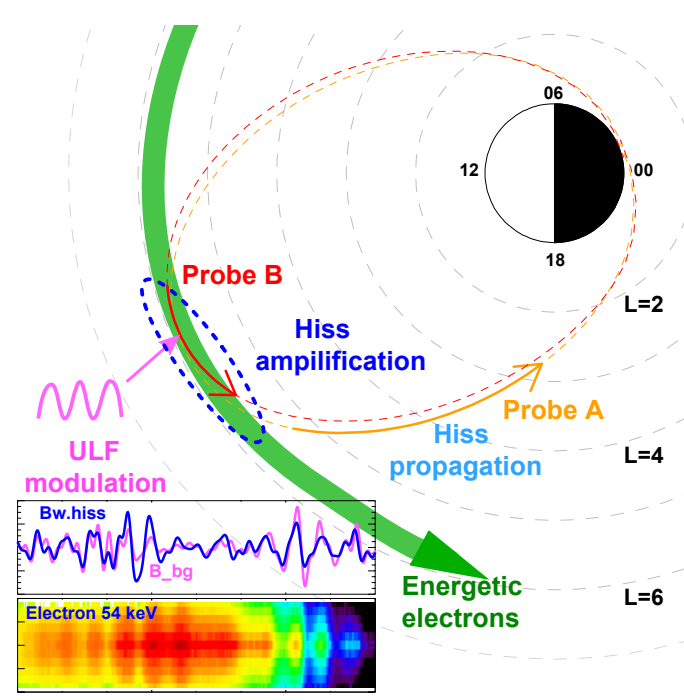

Figure 8. An illustration showing the energetic electron trajectory (green), ULF waves (pink) and hiss intensity modulation (blue). Injected electrons from the nightside drift to the post-noon sector (green arrow) in the outer plasmasphere where they provide a source of free energy for hiss wave generation in the outer plasmasphere. During the period of electron injection, electrons are modulated by ULF waves (magenta), which lead to the modulation of hiss wave amplification (blue), as observed by Van Allen Probe B. The hiss waves are probably generated in the outer plasmasphere, and then propagate into lower $L$ shells, as observed by Van Allen Probe A. 
trons resonant with chorus waves ( $\mathrm{Li}$ et al., 2011). Besides, the ULF wave-induced modulation of chorus could have an impact on electron precipitation leading to pulsating aurora (Jaynes et al., 2015). Similar modulations may also be captured in hiss wave intensity if hiss is locally amplified. However, different from chorus, plasmaspheric hiss waves are commonly known to be structureless (Thorne et al., 1973) and wave propagation is believed to be important for the measured hiss wave intensification (Bortnik et al., 2008, 2009; Chen et al., 2014). The hiss wave intensity is typically modulated by the variation in the background plasma density (Chen et al., 2012c). Nonetheless, our study showed the first evidence of the hiss wave modulation caused by modulated injected electrons due to ULF waves, clearly indicating that the hiss is locally amplified in the outer plasmasphere. It also provides an interesting link between the ULF waves and hiss waves which are in two distinct frequency ranges but both play important roles in radiation belt electron dynamics.

Data availability. The EMFISIS data are obtained from https:// emfisis.physics.uiowa.edu/data/index (last access: 24 April 2018), the MagEIS data are from http://www.rbsp-ect.lanl.gov/science/ DataDirectories.php (last access: 24 April 2018), and the EFW data are obtained from http://rbsp.space.umn.edu/data/rbsp/ (last access: 24 April 2018). The AE index is obtained from http://wdc.kugi. kyoto-u.ac.jp/aedir/index.html (last access: 24 April 2018).

Competing interests. The authors declare that they have no conflict of interest.

Acknowledgements. The work at Boston University is supported by the NASA grants NNX15AI96G, NNX17AG07G, and NNX17AD15G and the NSF grant AGS-1723342. The research at the University of Minnesota was supported by JHU/APL contract UMN 922613 under NASA contract JHU/APL NAS5-01072. We acknowledge the RBSP-ECT and EMFISIS funding provided by JHU/APL contract nos. 967399 and 921647 under NASA's prime contract no. NAS5-01072. We would like to thank Lei Dai and XuZhi Zhou for very helpful discussions in this study. We would like to acknowledge the EMFISIS data obtained from https://emfisis. physics.uiowa.edu/data/index, the MagEIS data obtained from http: //www.rbsp-ect.lanl.gov/science/DataDirectories.php, and the EFW data obtained from http://rbsp.space.umn.edu/data/rbsp/ (last access: 24 April 2018). We also thank the World Data Center for Geomagnetism, Kyoto for providing the AE index used in this study.

The topical editor, Minna Palmroth, thanks two anonymous referees for help in evaluating this paper.

\section{References}

Albert, J. M.: Evaluation of quasi-linear diffusion coefficients for whistler mode waves in a plasma with arbitrary density ratio, J. Geophys. Res., 110, A03218, https://doi.org/10.1029/2004JA010844, 2005.

Anderson, R. R. and Maeda, K.: VLF emissions associated with enhanced magnetospheric electrons, J. Geophys. Res., 82, 135146, https://doi.org/10.1029/JA082i001p00135, 1977.

Blake, J. B., Carranza, P. A., Claudepierre, S. G., Clemmons, J. H., Crain Jr., W. R., Dotan, Y., Fennell, J. F., Fuentes, F. H., Galvan, R. M., George, F. H., Henderson, M. G., Lalic, M., Lin, A. Y., Looper, M. D., Mabry, D. J., Mazur, J. E., McCarthy, B., Nguyen, C. Q., O’Brien, T. P., Perez, M. A., Redding, M. T., Roeder, J. L., Salvaggio, D. J., Sorensen, G. A., Spence, H. E., Yi, S., and Zakrzewski, M. P.: The Magnetic Electron Ion Spectrometer (MagEIS) Instruments Aboard the Radiation Belt Storm Probes (RBSP) spacecraft, Space. Sci. Rev., https://doi.org/10.1007/s11214-013-9991-8, 2013.

Bortnik, J., Thorne, R. M., and Meredith, N. P.: The unexpected origin of plasmaspheric hiss from discrete chorus emissions, $\mathrm{Na}-$ ture, 452, 62-66, https://doi.org/10.1038/nature06741, 2008.

Bortnik, J., Li, W., Thorne, R. M., Angelopoulos, V., Cully, C., Bonnell, J., Le Contel, O., and Roux, A.: An Observation linking the origin of plasmaspheric hiss to discrete chorus emissions, Science, 324, 775-778, https://doi.org/10.1126/science.1171273, 2009.

Breneman, A. W., Halford, A., Millan, R., McCarthy, M., Fennell, J., Sample, J., Woodger, L., Hospodarsky, G., Wygant, J. R., Cattell, C. A., Goldstein, J., Malaspina, D., and Kletzing, C. A.: Global-scale coherence modulation of radiation-belt electron loss from plasmaspheric hiss, Nature, 523, 193-195, https://doi.org/10.1038/nature14515, 2015.

Chen, M. W., Roeder, J. L., Fennell, J. F., Lyons, L. R., Lambour, R. L., and Schulz, M.: Proton ring current pitch angle distributions: Comparison of simulations with CRRES observations, J. Geophys. Res., 104, 17379-17389, 1999.

Chen, L., Bortnik, J., Li, W., Thorne, R. M., and Horne, R. B.: Modeling the properties of plasmaspheric hiss: 1. Dependence on chorus wave emission, J. Geophys. Res., 117, A05201, https://doi.org/10.1029/2011JA017201, 2012a.

Chen, L., Bortnik, J., Li, W., Thorne, R. M., and Horne, R. B.: Modeling the properties of plasmaspheric hiss: 2. Dependence on the plasma density distribution, J. Geophys. Res., 117, A05202, https://doi.org/10.1029/2011JA017202, 2012b.

Chen, L., Thorne, R. M., Li, W., Bortnik, J., Turner, D., and Angelopoulos, V.: Modulation of plasmaspheric hiss intensity by thermal plasma density structure, Geophys. Res. Lett., 39, L14103, https://doi.org/10.1029/2012GL052308, 2012c.

Chen, L., Thorne, R. M., Bortnik, J., Li, W., Horne, R., Reeves, G. D., Kletzing, C. A., Kurth, W. S., Hospodarsky, G. B., Spence, H. E., Blake, J. B., and Fennell, J. F.: Generation of unusually low frequency plasmaspheric hiss, Geophys. Res. Lett., 41, 57025709, https://doi.org/10.1002/2014GL060628, 2014.

Chen, X.-R., Zong, Q.-G., Zhou, X.-Z., Blake, J. B., Wygant, J. R., and Kletzing, C. A.: Van allen probes observation of a $360^{\circ}$ phase shift in the flux modulation of injected electrons by ULF waves, Geophys. Res. Lett., 44, 1614-1624, https://doi.org/10.1002/2016GL071252, 2016. 
Church, S. R. and Thorne, R. M.: On the origin of plasmaspheric hiss - Raypath integrated amplification, J. Geophys. Res., 88, 7941-7957, https://doi.org/10.1029/JA088iA10p07941, 1983.

Huang, C. Y., Goertz, C. K., and Anderson, R. R.: A theoretial study of plasmaspheric hiss generation, J. Geophys. Res., 88, 79277940, https://doi.org/10.1029/JA088iA10p07927, 1983.

Claudepierre, S. G., Mann, I. R., Takahashi, K., Fennell, J. F., Hudson, M. K., Blake, J. B., Roeder, J. L., Clemmons, J. H., Spence, H. E., Reeves, G. D., Baker, D. N., Funsten, H. O., Friedel, R. H. W., Henderson, M. G., Kletzing, C. A., Kurth, W. S., MacDowall, R. J., Smith, C. W., and Wygant, J. R.: Van Allen Probes observation of localized drift resonance between poloidal mode ultra-low frequency waves and $60 \mathrm{keV}$ electrons, Geophys. Res. Lett., 40, 4491-4497, https://doi.org/10.1002/grl.50901, 2013.

Dai, L., Takahashi, K., Wygant, J. R., Chen, L., Bonnell, J., Cattell, C. A., Thaller, S., Kletzing, C., Smith, C. W., MacDowall, R. J., Baker, D. N., Blake, J. B., Fennell, J., Claudepierre, S., Funsten, H. O., Reeves, G. D., and Spence, H. E.: Excitation of poloidal standing Alfven waves through drift resonance wave-particle interaction, Geophys. Res. Lett., 40, 4127-4132, https://doi.org/10.1002/grl.50800, 2013.

Green, J. L., Boardsen, S., Garcia, L., Taylor, W. W. L., Fung, S. F., and Reinisch, B. W.: On the origin of whistler mode radiation in the plasmasphere, J. Geophys. Res., 110, A03201, https://doi.org/10.1029/2004JA010495, 2005.

Hao, Y. X., Zong, Q.-G., Wang, Y. F., Zhou, X.-Z., Zhang, H., Fu, S. Y., Pu, Z. Y., Spence, H. E., Blake, J. B., Bonnell, J., Wygant, J. R., and Kletzing, C. A.: Interactions of energetic electrons with ULF waves triggered by interplanetary shock: Van Allen Probes observations in the magnetotail, J. Geophys. Res.Space, 119, 8262-8273, https://doi.org/10.1002/2014JA020023, 2014.

Jaynes, A. N., Lessard, M. R., Takahashi, K., Ali, A. F., Malaspina, D. M., Michell, R. G., Spanswick, E. L., Baker, D. N., Blake, J. B., Cully, C., Donovan, E. F., Kletzing, C. A., Reeves, G. D., Samara, M., Spence, H. E., and Wygant, J. R.: Correlated Pc4-5 ULF waves, whistler-mode chorus, and pulsating aurora observed by the Van Allen Probes and ground-based systems, J. Geophys. Res.-Space, 120, 8749-8761, https://doi.org/10.1002/2015JA021380, 2015.

Kennel, C. F. and Petschek, H. E.: Limit on stably trapped particle fluxes, J. Geophys. Res., 71, 1-28, https://doi.org/10.1029/JZ071i001p00001, 1966.

Kletzing, C. A., Kurth, W. S., Acuna, M., MacDowall, R. J., Torbert, R. B., Averkamp, T., Bodet, D., Bounds, S. R., Chutter, M., Connerney, J., Crawford, D., Dolan, J. S., Dvorsky, R., Hospodarsky, G. B., Howard, J., Jordanova, V., Johnson, R. A., Kirchner, D. L., Mokrzycki, B., Needell, G., Odom, J., Mark, D., Pfaff Jr., R., Phillips, J. R., Piker, C. W., Remington, S. L., Rowland, D., Santolik, O., Schnurr, R., Sheppard, D., Smith, C. W., Thorne, R. M., and Tyler, J.: The Electric and Magnetic Field Instrument Suite and Integrated Science (EMFISIS) on RBSP, Space Sci. Rev., 179, 127-181, https://doi.org/10.1007/s11214-013-9993-6, 2013.

Kurth, W. S., De Pascuale, S., Faden, J. B., Kletzing, C. A., Hospodarsky, G. B., Thaller, S., and Wygant, J. R.: Electron densities inferred from plasma wave spectra obtained by the Waves instrument on Van Allen Probes. J. Geophys. Res.-Space, 120, 904914, https://doi.org/10.1002/2014JA020857, 2015.
Li, L., Zhou, X.-Z., Zong, Q.-G., Rankin, R., Zou, H., Liu, Y., Chen, X.-R., and Hao, Y.-X.: Charged particle behavior in localized ultralow frequency waves: Theory and observations, Geophys. Res. Lett., 44, 5900-5908, https://doi.org/10.1002/2017GL073392, 2017.

Li, W., Thorne, R. M., Angelopoulos, V., Bonnell, J. W., McFadden, J. P., Carlson, C. W., LeContel, O., Roux, A., Glassmeier, K. H., and Auster, H. U.: Evaluation of whistler-mode chorus intensification on the nightside during an injection event observed on the THEMIS spacecraft, J. Geophys. Res., 114, A00C14, https://doi.org/10.1029/2008JA013554, 2009.

Li, W., Thorne, R. M., Bortnik, J., Nishimura, Y., and Angelopoulos, V.: Modulation of whistler mode chorus waves: 1 . Role of compressional Pc4-5 pulsations, J. Geophys. Res., 116, A06205, https://doi.org/10.1029/2010JA016312, 2011.

Li, W., Thorne, R. M., Bortnik, J., Reeves, G. D., Kletzing, C. A., Kurth, W. S., Hospodarsky, G. B., Spence, H. E., Blake, J. B., Fennell, J. F., Claudepierre, S. G., Wygant, J. R., and Thaller, S. A.: An unusual enhancement of low-frequency plasmaspheric hiss in the outer plasmasphere associated with substorm-injected electrons, Geophys. Res. Lett., 40, 37983803, https://doi.org/10.1002/grl.50787, 2013.

Li, W., Ma, Q., Thorne, R. M., Bortnik, J., Kletzing, C. A., Kurth, W. S., Hospodarsky, G. B., and Nishimura, Y.: Statistical properties of plasmaspheric hiss derived from Van Allen Probes data and their effects on radiation belt electron dynamics, J. Geophys. Res.-Space, 120, 3393-3405, https://doi.org/10.1002/2015JA021048, 2015a.

Li, W., Chen, L., Bortnik, J., Thorne, R. M., Angelopoulos, V., Kletzing, C. A., Kurth, W. S., and Hospodarsky, G. B.: First evidence for chorus at a large geocentric distance as a source of plasmaspheric hiss: Coordinated THEMIS and Van Allen Probes observation, Geophys. Res. Lett., 42, 241-248, https://doi.org/10.1002/2014GL062832, 2015b.

Lyons, L. R. and Thorne, R. M.: Equilibrium structure of radiation belt electrons, J. Geophys. Res., 78, 2142-2149, https://doi.org/10.1029/JA078i013p02142, 1973.

Lyons, L. R., Thorne, R. M., and Kennel, C. F.: Pitchangle diffusion of radiation belt electrons within the plasmasphere, J. Geophys. Res., 77, 3455-3474, https://doi.org/10.1029/JA077i019p03455, 1972.

Ma, Q., Li, W., Thorne, R. M., Bortnik, J., Reeves, G. D., Kletzing, C. A., Kurth, W. S., Hospodarsky, G. B., Spence, H. E., Baker, D. N., Blake, J. B., Fennell, J. F., Claudepierre, S. G., and Angelopoulos, V.: Characteristic energy range of electron scattering due to plasmaspheric hiss, J. Geophys. Res.-Space, 121, 1173711749, https://doi.org/10.1002/2016JA023311, 2016.

Mauk, B. H., Fox, N. J., Kanekal, S. G., Kessel, R. L., Sibeck, D. G., and Ukhorskiy, A.: Science Objectives and Rationale for the Radiation Belt Storm Probes Mission, Space Sci. Rev., 1-15, https://doi.org/10.1007/s11214-012-9908-y, 2012.

Meredith, N. P., Horne, R. B., and Anderson, R. R.: Substorm dependence of chorus amplitudes: Implications for the acceleration of electrons to relativistic energies,J. Geophys. Res., 106, 1316513178, https://doi.org/10.1029/2000JA900156, 2001.

Meredith, N. P., Horne, R. B., Clilverd, M. A., Horsfall, D., Thorne, R. M., and Anderson, R. R.: Origins of plasmaspheric hiss, J. Geophys. Res., 111, A09217, https://doi.org/10.1029/2006JA011707, 2006. 
Meredith, N. P., Horne, R. B., Glauert, S. A., and Anderson, R. R.: Slot region electron loss timescales due to plasmaspheric hiss and lightning generated whistlers, J. Geophys. Res., 112, A08214, https://doi.org/10.1029/2007JA012413, 2007.

Meredith, N. P., Horne, R. B., Glauert, S. A., Baker, D. N., Kanekal, S. G., and Albert, J. M.: Relativistic electron loss timescales in the slot region, J. Geophys. Res., 114, A03222, https://doi.org/10.1029/2008JA013889, 2009.

Meredith, N. P., Horne, R. B., Bortnik, J., Thorne, R. M., Chen, L., Li, W., and Sicard-Piet, A.: Global statistical evidence for chorus as the embryonic source of plasmaspheric hiss, Geophys. Res. Lett., 40, 2891-2896, https://doi.org/10.1002/grl.50593, 2013.

Ni, B., Bortnik, J., Thorne, R. M., Ma, Q., and Chen, L.: Resonant scattering and resultant pitch angle evolution of relativistic electrons by plasmaspheric hiss, J. Geophys. Res.-Space, 118, 7740-7751, https://doi.org/10.1002/2013JA019260, 2013.

Ni, B., Li, W., Thorne, R. M., Bortnik, J., Ma, Q., Chen, L., Kletzing, C. A., Kurth, W. S., Hospodarsky, G. B., Reeves, G. D., Spence, H. E., Blake, J. B., Fennell, J. F., and Claudepierre, S. G.: Resonant scattering of energetic electrons by unusual low-frequency hiss, Geophys. Res. Lett., 40, 3798-3803, https://doi.org/10.1002/2014GL059389, 2014.

Shi, R., Li, W., Ma, Q., Reeves, G. D., Kletzing, C. A., Kurth, W. S., Hospodarsky, G. B., Spence, H. E., Blake, J. B., Fennell, J. F., and Claudepierre, S. G.: Systematic evaluation of low-frequency hiss and energetic electron injections, J. Geophys. Res.-Space, 122, 10263-10274, https://doi.org/10.1002/2017JA024571, 2017.

Southwood, D. J. and Kivelson, M. G.: Charged particle behavior in low-frequency geomagnetic pulsations. I Transverse waves, J. Geophys. Res., 86 , 5643-5655, https://doi.org/10.1029/JA086iA07p05643, 1981.

Spence, H. E., Reeves, G. D., Baker, D. N., Blake, J. B., Bolton, M., Bourdarie, S., Chan, A. A., Claudepierre, S. G., Clemmons, J. H., Cravens, J. P., Elkington, S. R., Fennell, J. F., Friedel, R. H. W., Funsten, H. O., Goldstein, J., Green, J. C., Guthrie, A., Henderson, M. G., Horne, R. B., Hudson, M. K., Jahn, J.M., Jordanova, V. K., Kanekal, S. G., Klatt, B. W., Larsen, B. A., Li, X., MacDonald, E. A., Mann, I. R., Niehof, J., O’Brien, T. P., Onsager, T. G., Salvaggio, D., Skoug, R. M., Smith, S. S., Suther, L. L., Thomsen, M. F., and Thorne, R. M.: Science Goals and Overview of the Energetic Particle, Composition, and Thermal Plasma (ECT) Suite on NASA's Radiation Belt Storm Probes (RBSP) Mission, Space Sci. Rev., 179, 311-336, https://doi.org/10.1007/s11214-013-0007-5, 2013.

Summers, D., Ni, B., Meredith, N. P., Horne, R. B., Thorne, R. M., Moldwin, M. B., and Anderson, R. R.: Electron scattering by whistler-mode ELF hiss in plasmaspheric plumes, J. Geophys. Res., 113, A04219, https://doi.org/10.1029/2007JA012678, 2008.
Summers, D., Tang, R., and Thorne, R. M.: Limit on stably trapped particle fluxes in planetary magnetospheres, J. Geophys. Res., 114, A10210, https://doi.org/10.1029/2009JA014428, 2009.

Thorne, R. M., Smith, E. J., Burton, R. K., and Holzer, R. E.: Plasmaspheric hiss, J. Geophys. Res., 78, 1581-1596, https://doi.org/10.1029/JA078i010p01581, 1973.

Thorne, R. M., Church, S. R., and Gorney, D. J.: On the origin of plasmaspheric hiss - The importance of wave propagation and the plasmapause, J. Geophys. Res., 84, 5241-5247, https://doi.org/10.1029/JA084iA09p05241, 1979.

Wang, C., Zong, Q., Xiao, F., Su, Z., Wang, Y., and Yue, C.: The relations between magnetospheric chorus and hiss inside and outside the plasmasphere boundary layer: Cluster observation, J. Geophys. Res., 116, A07221, https://doi.org/10.1029/2010JA016240, 2011.

Wygant J. R., Bonnell, J. W., Goetz, K., Ergun, R. E., Mozer, F. S., Bale, S. D., Ludlam, M., Turin, P., Harvey, P. R., Hochmann, R., Harps, K., Dalton, G., McCauley, J., Rachelson, W., Gordon, D., Donakowski, B., Shultz, C., Smith, C., Diaz-Aguado, M., Fischer, J., Heavner, S., Berg, P., Malsapina, D. M., Bolton, M. K., Hudson, M., Strangeway, R. J., Baker, D. N., Li, X., Albert, J., Foster, J. C., Chaston, C. C., Mann, I., Donovan, E., Cully, C. M., Cattell, C. A., Krasnoselskikh, V., Kersten, K., Brenneman, A., and Tao, J. B.: The Electric Field and Waves Instruments on the Radiation Belt Storm Probes Mission, Space Sci. Rev., 179, 183-220, https://doi.org/10.1007/s11214-013-0013-7, 2013.

Zhou, X.-Z., Wang, Z.-H., Zong, Q.-G., Claudepierre, S. G., Mann, I. R., Kivelson, M. G., Angelopoulos, V., Hao, Y.X., Wang, Y.-F., and Pu, Z.-Y.: Imprints of impulseexcited hydromagnetic waves on electrons in the Van Allen radiation belts, Geophys. Res. Lett., 42, 6199-6204, https://doi.org/10.1002/2015GL064988, 2015.

Zhou, X.-Z., Wang, Z.-H., Zong, Q.-G., Rankin, R., Kivelson, M. G., Chen, X.-R., Blake, J. B., Wygant, J. R., and Kletzing, C. A.: Charged particle behavior in the growth and damping stages of ultralow frequency waves: Theory and Van Allen Probes observations, J. Geophys. Res.-Space, 121, 3254-3263, https://doi.org/10.1002/2016JA022447, 2016.

Zong, Q.-G., Zhou, X.-Z., Wang, Y. F., Li, X., Song, P., Baker, D. N., Fritz, T. A., Daly, P. W., Dunlop, M., and Pedersen, A.: Energetic electron response to ULF waves induced by interplanetary shocks in the outer radiation belt, J. Geophys. Res., 114, A10204, https://doi.org/10.1029/2009JA014393, 2009. 